\title{
Histoire des cuisiniers en France $\mathrm{XIXe-XXe}$ siècle
}

\author{
Alain Drouard
}

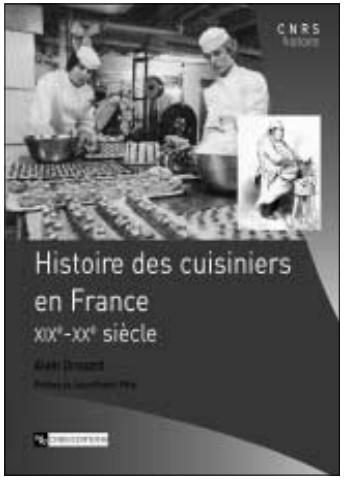

Si depuis une trentaine d'années la gloire et le prestige entourent les grands chefs français, l'histoire des cuisiniers ne se confond pas avec celle des cuisiniers les plus célèbres. Le monde des cuisines comprend en effet plusieurs catégories d' "ouvriers» et d'acteurs : à côté des cuisiniers de la restauration commerciale, il y a les cuisiniers de maison bourgeoise et les cuisiniers de la restauration collective. Si importantes qu'elles soient, ces distinctions n'ont pas empêché les cuisiniers de lutter ensemble pour changer leur statut dans la société.

Jusqu'à la Seconde Guerre mondiale, la condition de l'immense majorité des chefs et des cuisiniers était celle de domestiques ou d'employés. Dans les restaurants les chefs étaient placés sous l'autorité des restaurateurs, propriétaires des restaurants et des maîtres d'hôtel qui dans les salles étaient au contact direct de la clientèle. Les chefs et les cuisiniers ont été longs à s'affranchir de cette double tutelle et à gagner leur indépendance. Ils ont cherché à la fin du $\mathrm{XIX}^{\mathrm{e}}$ siècle à faire reconnaître leur compétence et leur qualification professionnelle puis ils sont devenus progressivement au $X X X^{e}$ siècle propriétaires de leurs restaurants. La médiatisation puis l'aventure de la Nouvelle Cuisine dans le dernier tiers du XXe siècle les ont mis en relation avec d'autres mondes - le show business, l'industrie et la finance - tandis que l'industrie agroalimentaire changeait complètement les conditions et les modalités d'exercice de leur métier. Quant aux cuisiniers de maisons bourgeoises, ils ont dû attendre les années soixante du $X X$ e siècle pour acquérir le statut de salarié qui n'est plus seulement celui des cuisiniers de la restauration collective mais celui de la majorité des cuisiniers en France.

Fondée sur des sources et des archives inédites, cette Histoire des cuisiniers en France aux $\mathrm{XIX}$ et $\mathrm{XX}$ siècles est celle d'un groupe, d'un métier, d'une profession en quête d'identité et de reconnaissance.

Normalien, agrégé d'histoire, docteur ès lettres, Alain DROUARD est directeur de recherche au CNRS (Centre Roland Mousnier, Paris). II s'est spécialisé dans l'histoire et la sociologie des sciences sociales. II travaille actuellement sur l'histoire de l'alimentation à l'époque contemporaine.

\section{Collection CNRS Histoire}

$17 \times 24$ - 192 p - 40 photos NB - br. $20 €$

\section{Pour trouver et commander nos ouvrages :}

LA LIBRAIRIE de CNRS ÉDITIONS, 151 bis, rue Saint-Jacques - 75005 PARIS

Tél. : 0153100505 - Télécopie : 0153100507 - Mél : lib.cnrseditions@wanadoo.fr

Site Internet : www.cnrseditions.fr

Frais de port par ouvrage : France : $5 €$ - Etranger : $5,5 €$

Pour plus de renseignements, n'hésitez pas à contacter

le Service clientèle de CNRS ÉDITIONS, 15, rue Malebranche - 75005 Paris

Tél : 0153102707 - Télécopie : 0153102727 - Mél : cnrseditions@cnrseditions.fr

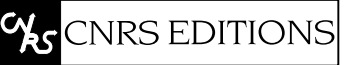

La référence du savoir 